K. YOSHIHARA

KODAI MATH. J.

2 (1979), 148-157

\title{
THE BOREL-CANTELLI LEMMA FOR STRONG MIXING SEQUENCES OF EVENTS AND THEIR APPLICATIONS TO LIL.
}

\author{
BY KeN-ICHI YOSHIHARA
}

1. Introduction. Let $\left\{\xi_{\jmath},-\infty<\jmath<\infty\right\}$ be a sequence of random variables which satisfy one of the following mixing conditions :

(I) the $\phi$-mixing condition, i.e.,

(1.1) $\quad \phi(n)=\sup _{k} \sup _{A \in M(-\infty, k), B \in M(k+n, \infty)}|P(A \cap B)-P(A) P(B)| / P(A) \downarrow 0 \quad(n \longrightarrow \infty)$

or

(II) the strong mixing (s. m.) condition, i.e.,

$$
\alpha(n)=\sup _{k} \sup _{A \in M(-\infty, k), B \in M(k+n, \infty)}|P(A \cap B)-P(A) P(B)| \downarrow 0 \quad(n \longrightarrow \infty)
$$

where $M(a, b)$ denotes the $\sigma$-algebra generated by $\xi_{a}, \cdots, \xi_{b}(a \leqq b)$.

By definition, it is obvious that if $\left\{\xi_{i}\right\}$ is $\phi$-mixing then $\left\{\xi_{i}\right\}$ is s.m.

Now, let $\left\{\bar{n}_{k}\right\}=\left\{n_{k}^{(i)}, i=1,2, k=1,2, \cdots\right\}$ be any sequence of positive integers such that

$$
n_{1}^{(1)} \leqq n_{1}^{(2)}<n_{2}^{(1)} \leqq n_{2}^{(2)}<\cdots<n_{k}^{(1)} \leqq n_{k}^{(2)}<\cdots
$$

For the sequence $\left\{\bar{n}_{k}\right\}$, we put

$$
\alpha_{i}^{*}\left(j:\left\{\bar{n}_{k}\right\}\right)=\sup _{A \in M\left(n_{\imath}^{(1)}, n_{\imath}^{(2)}\right), \boldsymbol{B} \in M\left(n_{i+j}^{(1)}, n_{l+j}^{(2)}\right.}|P(A \cap B)-P(A) P(B)|
$$

if $\left\{\xi_{i}\right\}$ is s.m. Then, it is clear that for any $i(\geqq 1)$ and $j(\geqq 1)$

$$
\alpha_{i}^{*}\left(\jmath:\left\{\bar{n}_{k}\right\}\right) \leqq \alpha\left(n_{\imath+\jmath}^{(1)}-n_{\imath}^{(2)}\right) .
$$

For brevity, we shall say that $\left\{A_{\imath}\right\} \quad\left(A_{i} \in M\left(n_{\imath}^{(1)}, n_{\imath}^{(2)}\right), i=1,2, \cdots\right)$ is $\alpha^{*}$-mixing with $\left\{\bar{n}_{k}\right\}$ if (1.5) holds.

In this paper, we shall prove the Borel-Cantelli lemma for such sequences of events and, using the result, establish some laws of iterated logarithm for mixing sequences of random variables.

Received November 2, 1977 
2. Some lemmas. In what follows, we shall agree to denote by the letter $K$ a quantity bounded in absolute value. For any random variable $\xi$, we put $\|\xi\|_{p}=\left\{E|\xi|^{p}\right\}^{p^{-1}}$ if the latter exists. The following two lemmas are known.

Lemma A. ([2] Theorem 17.2.1). Assume that condition (II) is satisfied and $\xi$ and $\eta$ are measurable over $M_{-\infty}^{k}$ and $M_{k+n}^{\infty}$ respectuvely. If $|\xi| \leqq C_{1}$ and $|\eta| \leqq C_{2}$ with probability one, then

$$
|E \xi \eta-E \xi E \eta| \leqq 4 C_{1} C_{2} \alpha(n) .
$$

Lemma B. ([1] Corollary to Lemma 2.1). Let $\xi$ and $\eta$ be the ones described in Lemma A. If $E|\xi|^{p}<\infty$ and $E|\eta|^{q}<\infty$ with $p>1, q>1$ and $p^{-1}+q^{-1}<1$, then

$$
|E \xi \eta-E \xi E \eta| \leqq 12\|\xi\|_{p}\|\eta\|_{q}\{\alpha(n)\}^{1-p^{-1}-q^{-1}} \text {. }
$$

LEMMA 1. Let $\left\{\xi_{i}\right\}$ be a s. m. sequence of random variables which satisfies the following conditions. For some $\delta>0$

(i) $E \xi_{\imath}=0$ and $E\left|\xi_{\imath}\right|^{2+\delta} \leqq K(\imath=1,2, \cdots)$, and

(ii) $\sum_{n=1}^{\infty}\{\alpha(n)\}^{\delta /(2+\delta)}<\infty$.

Then, for any $n(\geqq 1)$ and any $b(\geqq 0$ )

$$
\operatorname{Var}\left(\sum_{\imath=b+1}^{b+n} \xi_{\imath}\right) \leqq K \sum_{\imath=b+1}^{b+n}\left\|\xi_{i}\right\|_{2+\delta}^{2}
$$

and

$$
E\left(\max _{1 \leqq \jmath \leqq n}\left(\sum_{\imath=b+1}^{b+\jmath} \xi_{\imath}\right)^{2}\right) \leqq K\left\{\sum_{\imath=b+1}^{b+n}\left\|\xi_{i}\right\|_{2+\delta}^{2}\right\}\left(\log ^{2} 2 n\right) .
$$

Proof. Without loss of generality we assume that $b=0$. Since from Lemma B

So

$$
\begin{aligned}
\left|E \xi_{i} \xi_{\jmath}\right| & \leqq 12\left\|\xi_{i}\right\|_{2+\delta}\left\|\xi_{j}\right\|_{2+\delta}\{\alpha(\jmath-i)\}^{\delta /(2+\delta)} \\
& \leqq 6\left\{\left\|\xi_{i}\right\|_{2+\delta}^{2}+\left\|\xi_{j}\right\|_{2+\delta}^{2}\right\}\{\alpha(\jmath-i)\}^{\delta /(2+\delta)} \quad(j>i) .
\end{aligned}
$$

$$
\begin{aligned}
\operatorname{Var}\left(\sum_{\imath=1}^{n} \xi_{\imath}\right) & \leqq \sum_{i=1}^{n} E \xi_{\imath}^{2}+2 \sum_{i=1}^{n-1} \sum_{j=\imath+1}^{n}\left|E \xi_{i} \xi_{j}\right| \\
& \leqq \sum_{i=1}^{n} E \xi_{\imath}^{2}+12 \sum_{i=1}^{n-1} \sum_{\jmath=\imath+1}^{n}\left\{\left\|\xi_{i}\right\|_{2+\delta}^{2}+\left\|\xi_{j}\right\|_{2+\delta}^{2}\right\}\{\alpha(\jmath-i)\}^{\delta /(2+\delta)} \\
& \leqq \sum_{i=1}^{n} E \xi_{\imath}^{2}+12 \sum_{i=1}^{n}\left\|\xi_{i}\right\|_{2+\delta}^{2} \sum_{p=1}^{n-\imath}\{\alpha(p)\}^{\delta /(2+\delta)}+\sum_{j=2}^{n}\left\|\xi_{j}\right\|_{2+\delta}^{2} \sum_{q=1}^{j-1}\{\alpha(q)\}^{\delta /(2+\delta)} \\
& \leqq K \sum_{i=1}^{n}\left\|\xi_{i}\right\|_{2+\delta}^{2} .
\end{aligned}
$$

Thus, we have (2.3). (2.4) is obtained from Theorem $F$ in [4] and (2.3). 
3. The Borel-Cantelli lemmas for $\alpha^{*}$-mixing sequences of events.

For the $\alpha^{*}$-mixing sequence of events the following theorem holds.

THEOREM 1. Let $\left\{\xi_{i}\right\}$ be a (not necessarily structly stationary) s. m. sequence with $\sum\{\alpha(n)\}^{\delta /(2+\delta)}<\infty$ for some $\delta>0$. Let $\left\{\bar{n}_{k}\right\}$ be an arbitrary sequence of positvve integers satisfying (1.3). Suppose that the sequence $\left\{A_{i}\right\}$ of events be $\alpha^{*}$-mixing with $\left\{\bar{n}_{k}\right\}$. If for some $a(0<a<(2+\delta) /(2+2 \delta))$

$$
\liminf _{n \rightarrow \infty} n^{a} P\left(A_{n}\right)>0
$$

then

$$
P\left(\bigcap_{n=1}^{\infty} \bigcup_{k=n}^{\infty} A_{k}\right)=1 .
$$

Thus, there occur with probability one infinitely many of the events $A_{n}$,

Proof. We shall use Révész's method in [8]. Let $\zeta_{n}$ be the indicator function of $A_{n}$, i. e.,

$$
\zeta_{n}=\zeta_{n}(\omega)=\left\{\begin{array}{lll}
1 & \text { if } & \omega \in A_{n}, \\
0 & \text { if } & \omega \notin A_{n} .
\end{array}\right.
$$

Then, $\left\{\zeta_{n}\right\}$ is a s. m. sequence of random variables and by the Chebyshev inequality

$$
\begin{aligned}
D_{n} & =P\left(\sum_{\imath=1}^{n} \zeta_{\imath} \leqq \frac{1}{2} \sum_{\imath=1}^{n} P\left(A_{\imath}\right)\right) \\
& \leqq P\left(\left|\sum_{\imath=1}^{n} \zeta_{\imath}-\sum_{\imath=1}^{n} P\left(A_{\imath}\right)\right| \geqq \varepsilon \sum_{\imath=1}^{n} P\left(A_{\imath}\right)\right) \\
& \leqq \frac{\operatorname{Var}\left(\sum_{\imath=1}^{n} \zeta_{\imath}\right)}{\varepsilon^{2}\left\{\sum_{\imath=1}^{n} P\left(A_{\imath}\right)\right\}^{2}}
\end{aligned}
$$

where $\varepsilon(0<\varepsilon<1 / 2)$ is arbitrary. Since by Lemma 1

$$
\begin{aligned}
\operatorname{Var}\left(\sum_{i=1}^{n} \zeta_{\imath}\right) & \leqq K \sum_{i=1}^{n}\left\|\zeta_{i}-P\left(A_{\imath}\right)\right\|_{2+\delta}^{2} \\
& \leqq K \sum_{i=1}^{n}\left\{E\left(\zeta_{i}-P\left(A_{\imath}\right)\right)^{2}\right\}^{2 /(2+\delta)} \leqq K \sum_{i=1}^{n}\left\{P\left(A_{\imath}\right)\right\}^{2 /(2+\delta)},
\end{aligned}
$$

so

$$
D_{n} \leqq \frac{K \sum_{\imath=1}^{n}\left\{P\left(A_{\imath}\right)\right\}^{2 /(2+\delta)}}{\varepsilon^{2}\left\{\sum_{\imath=1}^{n} P\left(A_{\imath}\right)\right\}^{2}} .
$$

Hence, from (3.1), we have 


$$
D_{n} \longrightarrow 0 \quad(n \longrightarrow \infty) \text {. }
$$

Thus, there exists a sequence $\left\{k_{j}\right\}$ of the integers such that

$$
\sum_{j=1}^{\infty} D_{k_{\jmath}}<\infty
$$

It follows from the original form of the Borel-Cantelli lemma that there occur with probability one only finitely many of the events

$$
B_{j}=\left\{\sum_{i=1}^{k} \zeta_{\imath} \leqq \frac{1}{2} \sum_{i=1}^{k} P\left(A_{\imath}\right)\right\} \text {. }
$$

Since the sequence $\left\{\sum_{\imath=1}^{k} \zeta_{\imath}: k=1,2, \cdots\right\}$ is non-decreasing, so we have the desired conclusion.

THEOREM 2. Let $\left\{\xi_{i}\right\}$ be a (not necessarily structly stationary) s.m. sequence of random varnables with $\alpha(n)$. Let $\left\{\bar{n}_{k}\right\}$ be an arbitrary sequence of positvve integers which satısfy (1.3) and for which

$$
\sum_{i=1}^{\infty} \sum_{\jmath=\imath+1}^{\infty} \alpha_{i}^{*}\left(\jmath:\left\{\bar{n}_{k}\right\}\right)<\infty
$$

holds. Suppose that the sequence $\left\{A_{\imath}\right\}$ of events be $\alpha^{*}$-mixing with $\left\{\bar{n}_{k}\right\}$. Then

$$
P\left(\bigcap_{n=1}^{\infty} \bigcup_{\imath=n}^{\infty} A_{\imath}\right)=1 \quad(\text { or } 0)
$$

according as

$$
\sum_{n=1}^{\infty} P\left(A_{n}\right)=\infty \quad(\text { or }<\infty)
$$

Remark. It is obvious that if condition (II) is satisfied, then there always exists a sequence $\left\{\bar{n}_{k}\right\}$ satisfying (3.3).

Proof of Theorem 2. From the original form of Borel-Cantelli lemma, it is enough to prove that $\sum_{n=1}^{\infty} P\left(A_{n}\right)=\infty$ implies $P\left(\bigcap_{n=1}^{\infty} \bigcup_{l=n}^{\infty} A_{\imath}\right)=1$. As before, let $\zeta_{n}$ be the indicator function of $A_{n}$. Then, from Lemma A (with $C_{1}=C_{2}=1$ ) and (3.3)

$$
\begin{aligned}
\operatorname{Var}\left(\sum_{\imath=1}^{n} \zeta_{\imath}\right) & =\sum_{\imath=1}^{n} \operatorname{Var}\left(\zeta_{\imath}\right)+2 \sum_{i=1}^{n-1} \sum_{\jmath=\imath+1}^{n} E\left(\zeta_{\imath}-P\left(A_{\imath}\right)\right)\left(\zeta_{\jmath}-P\left(A_{\jmath}\right)\right) \\
& \leqq \sum_{\imath=1}^{n} \operatorname{Var}\left(\zeta_{\imath}\right)+4 \sum_{\imath=1}^{n-1} \sum_{\jmath=\imath+1}^{n} \alpha_{i}^{*}\left(\jmath:\left\{\bar{n}_{k}\right\}\right) \\
& \leqq \sum_{\imath=1}^{n} P\left(A_{\imath}\right)+K .
\end{aligned}
$$

Thus, for any $\varepsilon(0<\varepsilon<1)$ 


$$
P\left(\sum_{\imath=1}^{n} \zeta_{i}<\frac{1}{2} \sum_{\imath=1}^{n} P\left(A_{\imath}\right)\right) \leqq \frac{\operatorname{Var}\left(\sum_{\imath=1}^{n} \zeta_{\imath}\right)}{\varepsilon^{2}\left\{\sum_{\imath=1}^{n} P\left(A_{\imath}\right)\right\}^{2}} \leqq \frac{\sum_{i=1}^{n} P\left(A_{\imath}\right)+K}{\varepsilon^{2}\left\{\sum_{\imath=1}^{n} P\left(A_{\imath}\right)\right\}^{2}} \longrightarrow 0 \quad(n \longrightarrow \infty)
$$

if $\sum_{\imath=1}^{n} P\left(A_{\imath}\right) \rightarrow \infty(n \rightarrow \infty)$

The rest of the proof can be carried out by the analogous method to the proof of Theorem 1 and so is omitted.

4. Some applications to LIL for mixing sequences. Let $\left\{\xi_{i}\right\}$ be a mixing sequence of random variables centered at expectations with finite variances. Put

$$
s_{n}^{2}=E\left(\sum_{j \leqq n} \xi_{j}\right)^{2} .
$$

In this section we assume that

$$
\begin{gathered}
\frac{s_{n+1}}{s_{n}} \longrightarrow 1 \quad(n \longrightarrow \infty), \\
0<\liminf _{n \rightarrow \infty} \frac{s_{n}^{2}}{n} \leqq \limsup _{n \rightarrow \infty} \frac{s_{n}^{2}}{n}<\infty
\end{gathered}
$$

and

$$
s_{m, n}^{2}=E\left(\sum_{\jmath=m+1}^{n} \xi_{\jmath}\right)^{2}=\left(s_{n}^{2}-s_{m}^{2}\right)(1+o(1)) \quad(\text { as } n-m \rightarrow \infty) .
$$

We consider the following conditions:

(A) $\left\{\xi_{n}\right\}$ satisfies condition (I) with $\phi(n)=O\left(n^{-1}\right)$, and

(B) $\left\{\xi_{n}\right\}$ satisfies condition (II) with $\alpha(n)=O\left(n^{-1}\right)$.

THEOREM 3. Let $\left\{\xi_{i}\right\}$ be a mixing sequence satisfying (4.2)-(4.4) and one of Condition (A) or (B). Suppose that for some $\eta>0$ uniformly in $m$ and $x$

$$
P\left(s_{m, n}^{-1} \sum_{j=m+1}^{n} \xi_{j}<x\right)=(2 \pi)^{-1 / 2} \int_{-\infty}^{x} e^{-1 / 2 t^{2}} d t+O\left(\left(\log s_{m, n}\right)^{-1-\eta}\right)
$$

and that for some constants $C>0,0<d_{n}=O\left(\left(\log \log s_{n}\right)^{1 / 2}\right)$ and $\beta$ sufficiently large

$$
P\left(\max _{1 \leqq j \leqq n} \sum_{i=1}^{j} \xi_{\imath}>\beta\right) \leqq C P\left(\sum_{\jmath \leqq n} \xi_{j}>\beta-d_{n} s_{n}\right) .
$$

Moreover, suppose that (4.6) holds with $\xi_{n}$ replaced by $-\xi_{n}$. Then

$$
P\left(\limsup _{n \rightarrow \infty}\left(2 s_{n}^{2} \log \log s_{n}^{2}\right)^{-1 / 2} \sum_{j \leqq n} \xi_{\jmath}=1\right)=1 .
$$

Proof. We shall prove Theorem 3 in the case where $\left\{\xi_{\imath}\right\}$ satisfies condition (B). The assertion (4.7) will be proved if we show that for any $\varepsilon>0$ 


$$
P\left(S_{n}>(1+\varepsilon) b(n) \text { i. o. }\right)=0
$$

and

$$
P\left(S_{n}>(1-\varepsilon) b(n) \text { i. o. }\right)=1
$$

where

$$
b(n)=\left(2 s_{n}^{2} \log \log s_{n}^{2}\right)^{1 / 2} .
$$

Thus, to prove the theorem it is enough to show (4.9) since (4.8) is proved by the usual method (cf. [6] and Theorem 0 in [7]).

Let $c>1$ be an arbitrarily given number. We choose $n_{k}^{(1)}$ and $n_{k}^{(2)}$ as follows:

$$
\begin{gathered}
n_{k}^{(2)}=\text { the largest integer } n \text { such that } s_{n} \leqq c^{k}, \\
n_{k}^{(1)}=\text { the largest integer } n \text { such that } s_{n} \leqq c^{k-1}+c^{k / 2} .
\end{gathered}
$$

For any $\gamma(0<\gamma<1)$ put

$$
B_{k}=B_{k}(\gamma)=\left\{S_{n_{k}^{(2)}}-S_{n_{k}^{(1)}}>(1-\gamma) a\left(n_{k}\right)\right\}
$$

where

$$
a\left(n_{k}\right)=\left\{2\left(s_{n_{k}^{2}(2)}^{2}-s_{n_{k}}^{2(1)}\right) \log \left(s_{n_{k}^{(2)}}^{2}-s_{n_{k}}^{2}(1)\right)\right\}^{1 / 2} .
$$

Then, $\left\{B_{k}\right\}$ is $\alpha^{*}$-mixing with $\left\{\bar{n}_{k}\right\}=\left\{n_{k}^{(1)}, n_{k}^{(2)}\right\}$. Since by definition and (4.3)

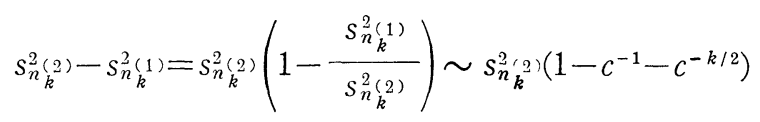

for sufficiently large $k$, so from (4.5) we get

$$
\sum_{k} P\left(B_{k}\right)>\sum_{k}\left\{\log \left(s_{n_{k}^{(2)}}^{2}-s_{n_{k}^{(1)}}^{2}\right)\right\}^{-(1+\mu)(1-\gamma)^{2}}>K \sum_{k} k^{-(1+\mu)(1-\gamma)^{2}}=\infty
$$

where $\mu$ is an arbitrary positive number such that $(1+\mu)(1-\gamma)^{2}<1$.

On the other hand

$$
\begin{aligned}
& \sum_{i=1}^{\infty} \sum_{j=1}^{\infty} \alpha_{i}^{*}\left(\jmath:\left\{\bar{n}_{k}\right\}\right)=\sum_{\imath=1}^{\infty} \sum_{j=1}^{\infty} \alpha\left(n_{\imath+j}^{(1)}-n_{\imath}^{(2)}\right) \\
\leqq & K \sum_{i=1}^{\infty} \sum_{j=1}^{\infty} \frac{1}{n_{\imath+j}^{(1)}-n_{\imath}^{(2)}} \leqq K \sum_{i=1}^{\infty} \sum_{j=1}^{\infty} \frac{1}{c^{\imath}\left(c^{\jmath-1}+c^{(\jmath-\imath) / 2-1}\right)}<\infty .
\end{aligned}
$$

Hence, by Theorem 2 we have

$$
P\left(B_{k}(\gamma) \text { i. o. }\right)=1
$$

for any $\gamma$ such that $0<\gamma<1$.

Now, we use the method of the proof of Theorem 1 in [6]. From (4.8) it follows that

$$
\left|S_{n}(\omega)\right| \leqq 2 b(n) \quad \text { for } n>n_{0}(\omega) \quad \text { a.s. }
$$


Taking (4.10) into account, we find

$$
\begin{aligned}
(1-\gamma) & a\left(\bar{n}_{k}\right)-2 b\left(n_{k-1}^{(2)}\right) \\
& \sim\left\{(1-\gamma)\left(1-c^{-1}\right)^{1 / 2}-2 c^{-1 / 2}\right\} b\left(n_{k}^{(2)}\right) .
\end{aligned}
$$

Since $\varepsilon>0$ is arbitrary, we may choose $\gamma>0$ and $c>1$ so that

$$
(1-\gamma)\left(1-c^{-1}\right)^{1 / 2}-2 c^{-1 / 2}>1-\varepsilon .
$$

Using (4.13), we find

$$
\begin{aligned}
P\left(S_{n_{k}^{(2)}}\right. & \left.>(1-\varepsilon) b\left(n_{k}^{(2)}\right) \text { i. o. }\right) \\
& \geqq P\left(S_{n_{k}^{(2)}}>(1-\gamma) a\left(n_{k}\right)-2 b\left(n_{k-1}^{(2)}\right) \text { i. o. }\right) \\
& \geqq P\left(B_{k}(\gamma) \text { i. o. }\right)=1 .
\end{aligned}
$$

From this (4.9) follows and the theorem is proved in the case where condition (B) holds. The proof in the case where condition (A) holds is similar and so is omitted.

Remark 1. It is obvious from the above proof that the mixing condition in (A) (or (B)) may be weakened as $\phi(n)=O\left(n^{-a}\right)$ (or $\alpha(n)=O\left(n^{-a}\right)$ ) for some $a(0<$ $a<1)$.

Remark 2. It is obvious that Theorem 3 holds if (4.6) is replaced by the condition

$$
P\left(\max _{1 \leqq J \leqq n}\left|\sum_{\imath=1}^{j} \xi_{\imath}\right|>\varepsilon b\left(s_{n}\right)\right)=O\left(\left(\log s_{n}\right)^{-1-\eta}\right)
$$

where $\eta$ and $\varepsilon$ are arbitrary positive numbers.

For the s. m. sequence $\left\{\xi_{i}\right\}$ with coefficient $\alpha(n)$, condition (4.15) is satisfied, if (4.5) holds and there exists a function $r=r(n)$ such that $r(n) \rightarrow \infty$ and

$$
\begin{aligned}
& \max \left\{\sum_{\imath=0}^{k-2} P\left(\max _{1 \leqq \jmath \jmath r}\left|S_{(i+2) r}-S_{\imath r+j}\right| \geqq \varepsilon b\left(s_{n}\right)\right),\right. \\
&\left.P\left(\max _{1 \leqq \jmath \geqq r}\left|S_{n}-S_{n-\jmath}\right| \geqq \varepsilon b\left(s_{n}\right)\right), k \alpha(r)\right\}=O\left(\left(\log s_{n}\right)^{-1-\eta}\right)
\end{aligned}
$$

for any $\varepsilon(0<\varepsilon<(c-1) / c)$ where $c>1$ is an arbitrarily fixed number and $k=[n / r]$. In fact, for any $c>1$, let

$$
E_{j}=\left\{\left|S_{\imath}\right|<c b\left(s_{n}\right), \imath<\jmath:\left|S_{j}\right| \geqq c b\left(s_{n}\right)\right\} \quad(\jmath=1, \cdots, n) .
$$

It follows from (II) that for any $\varepsilon(0<\varepsilon<1)$

$$
I=P\left(\max _{1 \leqq \jmath \geqq n}\left|S_{j}\right| \geqq c b\left(s_{n}\right)\right)=P\left(\bigcup_{\jmath=1}^{n} E_{\jmath}\right)
$$




$$
\begin{aligned}
\leqq & P\left(\left|S_{n}\right| \geqq c(1-\varepsilon) b\left(s_{n}\right)\right)+\sum_{\imath=0}^{k-2} P\left(\bigcup_{\jmath=1}^{r}\left[E_{\imath r+j} \cap\left\{\left|S_{n}-S_{\imath r+\jmath}\right| \geqq \varepsilon c b\left(s_{n}\right)\right\}\right]\right) \\
& +\sum_{l=(k-1) r+1}^{n} P\left(E_{l} \cap\left\{\left|S_{n}-S_{l}\right| \geqq \varepsilon b\left(s_{n}\right)\right\}\right) \\
\leqq & P\left(\left|S_{n}\right| \geqq c(1-\varepsilon) b\left(s_{n}\right)\right)+\sum_{\imath=0}^{k-2} P\left(\left(\bigcup_{\jmath=1}^{r} E_{\imath r+\jmath}\right) \cap\left\{\left|S_{n}-S_{(i+2) r}\right| \geqq \frac{\varepsilon}{2} b\left(s_{n}\right)\right\}\right) \\
& +\sum_{\imath=0}^{k-2} P\left(\bigcup_{\jmath=1}^{r}\left[E_{\imath r+j} \cap\left\{\left|S_{(i+2) r}-S_{\imath r+\jmath}\right| \geqq \frac{\varepsilon}{2} b\left(s_{n}\right)\right\}\right]\right) \\
& +\sum_{l=(k-1) r+1}^{n} P\left(E_{l} \cap\left\{\left|S_{n}-S_{l}\right| \geqq \varepsilon b\left(s_{n}\right)\right\}\right) \\
\leqq & P\left(\left|S_{n}\right| \geqq c(1-\varepsilon) b\left(s_{n}\right)\right)+\sum_{\imath=0}^{k-2} P\left(\bigcup_{\jmath=1}^{r} E_{\imath r+\jmath}\right) P\left(\left|S_{n}-S_{(i+2) r}\right| \geqq \frac{\varepsilon}{2} b\left(s_{n}\right)\right) \\
& +k \alpha(r)+\sum_{\imath=1}^{k-2} P\left(\max _{1 \leqq \jmath \leq r}\left|S_{(\imath+2) r}-S_{\imath r+j}\right| \geqq \frac{\varepsilon}{2} b\left(s_{n}\right)\right) \\
& +P\left(\max _{(k-1) r+1 \leqq \jmath \leqq n-1}\left|S_{n}-S_{j}\right| \geqq \varepsilon b\left(s_{n}\right)\right) .
\end{aligned}
$$

Since it follows from (4.1) and (4.4) that for any $i(0 \leqq \imath \leqq k-1)$

$$
P\left(\left|S_{n}-S_{(i+2) r}\right| \geqq \varepsilon b\left(s_{n}\right)\right) \leqq \frac{S_{(\imath+2) r, n}^{2}}{\varepsilon^{2} b^{2}\left(s_{n}\right)} \longrightarrow 0 \quad(n \longrightarrow \infty),
$$

so for all $n$ sufficiently large

$$
P\left(\left|S_{n}-S_{(i+2) r}\right| \geqq \varepsilon b\left(s_{n}\right)\right) \leqq \frac{1}{2} .
$$

Hence from (4.16)

$$
I \leqq O\left(\left(\log s_{n}\right)^{-1-\eta}\right)+\frac{1}{2} \sum_{\imath=0}^{k-2} P\left(\bigcup_{\jmath=1}^{r} E_{\imath r+\jmath}\right)=O\left(\left(\log s_{n}\right)^{-1-\eta}\right)+\frac{1}{2} I
$$

and so

$$
I=O\left(\left(\log s_{n}\right)^{-1-\eta}\right)
$$

5. Sufficient conditions of LIL for mixing sequences. In this section, we assume that $\left\{\xi_{i}\right\}$ is a (not necessarily strictly stationary) mixing sequence of random variables with $E \xi_{i}=0(\imath=1,2, \cdots)$. We shall consider some conditions:

Condition (5. a): $\left\{\xi_{i}\right\}$ is $\phi$-mixing with $\Sigma \phi^{1 / 2}(n)<\infty$, and

$$
\int_{|x|>N} x^{2} d F_{\imath}(x) \leqq K(\log N)^{-5}
$$

uniformly in $\imath$, where $F_{\imath}$ is the $d f$ of $\xi_{\imath}$.

Condition (5. b): $\left\{\xi_{\imath}\right\}$ is $\phi$-mixing and for some $\delta>0$ the followings hold;

$1^{0} E\left|\xi_{\imath}\right|^{2+\delta} \leqq K \quad$ uniformly in $\imath$, 


$$
2^{0} \phi(n)=O\left(n^{-1-\varepsilon}\right) \quad \text { for some } \varepsilon>(1+\delta)^{-1} .
$$

Condition (5. c): $\left\{\xi_{i}\right\}$ is s. m. with $\alpha(n)=O\left(n^{-1-\varepsilon}\right)$ for some $\varepsilon>0$ and uniformly bounded with probability one, i. e., $\left|\xi_{\imath}\right| \leqq K(i=1,2, \cdots)$.

Condition (5. d): $\left\{\xi_{i}\right\}$ is s. m. and for some $\delta>0$ the followings hold:

$$
\begin{array}{ll}
1^{0} \quad E\left|\xi_{\imath}\right|^{2+\delta} \leqq K \quad \text { uniformly in } i, \\
2^{0} \sum_{n=1}^{\infty}\{\alpha(n)\}^{\delta^{\prime} /\left(2+\delta^{\prime}\right)}<\infty \quad \text { for some } 0<\delta^{\prime}<\delta .
\end{array}
$$

LEMMA 3. If the mixing sequence $\left\{\xi_{i}\right\}$ satisfies (4.2)-(4.4) and one of conditrons (5. a)-(5. d), then (4.15) is satisfied.

Proof. Let the conditions (4.2)-(4.4) and (5. d) be satisfied. Put $r=\left[s_{n}^{1 / 2}\right]$. Then, from Lemma 2, (4.2) and (4.3) it follows that

$$
P\left(\max _{(k-1) r+1 \leqq j \leqq n-1}\left|S_{n}-S_{j}\right| \geqq \varepsilon b\left(s_{n}\right)\right) \leqq \frac{K s_{(k-1) r, n}^{2}\left(\log ^{2} 4 r\right)}{\varepsilon^{2} s_{n}^{2}}=o\left(s_{n}^{-r}\right)
$$

for some $\gamma>0$.

Next, for $N=s_{n}^{1 / 2}$ let

$$
f_{N}\left(\xi_{\imath}\right)=\left\{\begin{array}{ll}
\xi_{\imath} & \left(\left|\xi_{\imath}\right| \leqq N\right) \\
0 & \left(\left|\xi_{\imath}\right|>N\right)
\end{array} \quad(i=1, \cdots, n)\right.
$$

and $\bar{f}_{N}\left(\xi_{\imath}\right)=\xi_{i}-f_{N}\left(\xi_{\imath}\right)$. As

$$
\left|\sum_{p=\imath r+1}^{(i+2) r}\left(f_{N}\left(\xi_{p}\right)-E f_{N}\left(\xi_{p}\right)\right)\right| \leqq 4 r N<\frac{\varepsilon}{2} b\left(s_{n}\right)
$$

for all $n$ sufficiently large, so by Lemma 2

$$
\begin{aligned}
& P\left(\max _{1 \leqq \jmath \leqq r}\left|S_{(i+2) r}-S_{\imath r+j}\right| \geqq \varepsilon b\left(s_{n}\right)\right) \\
& \leqq P\left(\max _{1 \leqq \jmath \leqq r}\left|\sum_{p=\imath r+j}^{(i+2) r}\left(\bar{f}_{N}\left(\xi_{p}\right)-E \bar{f}_{N}\left(\xi_{p}\right)\right)\right| \geqq \frac{\varepsilon}{2} b\left(s_{n}\right)\right) \\
&+P\left(\sum_{p=\imath r+1}^{(i+2) r}\left|f_{N}\left(\xi_{p}\right)-E f_{N}\left(\xi_{p}\right)\right| \geqq \frac{\varepsilon}{2} b\left(s_{n}\right)\right) \\
&= P\left(\max _{1 \leqq j \leqq r}\left|\sum_{p=i r+j}^{(i+2) r}\left(\bar{f}_{N}\left(\xi_{p}\right)-E \bar{f}_{N}\left(\xi_{p}\right)\right)\right| \geqq \frac{\varepsilon}{2} b\left(s_{n}\right)\right) \\
& \leqq \frac{K\left(\log ^{2} 4 r\right)}{\varepsilon^{2} b^{2}\left(s_{n}\right)} \sum_{p=\imath r+1}^{(i+2) r}\left\{E\left|\bar{f}_{N}\left(\xi_{p}\right)-E \bar{f}_{N}\left(\xi_{p}\right)\right|^{2+\delta^{\prime}}\right\}^{2 /\left(2+\delta^{\prime}\right)} \\
& \leqq \frac{K r\left(\log ^{2} 4 r\right)}{\varepsilon^{2} b^{2}\left(s_{n}\right)}\left\{N^{-\left(\delta-\delta^{\prime}\right)}\right\}^{2 /(2+\delta)} \quad \max _{\imath r+1 \leqq p \leqq(i+2) r}\left\{E\left|\bar{f}_{N}\left(\xi_{p}\right)-E \bar{f}_{N}\left(\xi_{p}\right)\right|^{2+\delta}\right\}^{2 / 2+\delta} \\
& \leqq \frac{K r\left(\log ^{2} 4 r\right)}{\varepsilon^{2} b^{2}\left(s_{n}\right)} s_{n}^{-\left(\delta-\delta^{\prime}\right) /\left(2+\delta^{\prime}\right)}, \quad(\imath=0, \cdots, k-2)
\end{aligned}
$$


and consequently

$$
\sum_{\imath=0}^{k-2} P\left(\max _{1 \leqq j \leqq r}\left|S_{(i+2) r}-S_{\imath r+j}\right| \geqq \varepsilon b\left(s_{n}\right)\right)=o\left(s_{n}^{-r}\right)
$$

for some $\gamma>0$. Hence (4.16) holds.

The proofs in other cases are similar and so are omitted.

Now, we have laws of the iterated logarithm for mixing sequences, which are some extensions of the results in [6].

THEOREM 4. If the mixing sequence $\left\{\xi_{i}\right\}$ satisfies (4.2)-(4.4) and one of (5. a)(5. d), then (4.7) holds.

Proof. By Lemma 3, to prove the theorem it is enough to show (4.5) in each case. But the proofs are almost identical with those of Theorems 1-4 in [5] and therefore are omitted.

\section{REFERENCES}

[1] Davydov, Yu. A. (1968) - Convergence of distributions generated by stationary stochastic processes. Theory Probab. Appli. 13, 691-696.

[2] Ibragimov, I. A., LinNik, Yu. V. (1971): Independent and stationary sequences of random variables. Groningen-Noordhoff.

[3] Iosifescu, M. (1968): The law of the iterated logarithm for a class of dependent random variables. Theory Probab. Appli. 13, 304-313.

[4] MoRICZ, F. (1976): Moment inequalities and the strong laws of large numbers. Z. Wahrscheinlichkeitstheorie verw. Geb. 35, 297-314.

[5] Oodaira, H., Yoshinara, K. (1971): The law of the iterated logarithm for stationary processes satisfying mixing conditions. Kōdai Math. Sem. Rep. 23, 311-334.

[6] Petrov, V.V. (1966): On a relation between an estımate of the remainder in the central limit theorem and the law of the iterated logarithm. Theory Probab. Appli. 11, 454-458.

[7] Philipp, W. (1969): The law of the iterated logarithm for mixing stochastic processes. Ann. Math. Statist. 40, 1985-1991.

[8] RÉvész, P. (1968): The laws of the large numbers. New York Academic Press.

[9] RÉvész, P. (1972): The law of the iterated logarithm for multiplicative systems. Indiana Univ. Math. J. 21, 557-564. 